\title{
Efficacy and Safety of a Lercanidipine/Enalapril Fixed-Dose Combination in Hypertensive Patients in Portugal
}

\author{
João Maldonado • Telmo Pereira • Alfredo Tavares
}

Published online: 16 May 2014

(C) The Author(s) 2014. This article is published with open access at Springerlink.com

\begin{abstract}
Background Fixed-dose combinations of hypertensive drugs have been advocated as a suitable option for hypertensive patients who require two or more drugs to achieve blood pressure (BP) targets.

Objectives Our objective was to assess the efficacy and safety of lercanidipine/enalapril in clinical practice.

Methods This observational study collected data for patients with hypertension treated by 46 specialists at clinics across Portugal with lercanidipine/enalapril (10/ $20 \mathrm{mg}$ ). The primary outcome measure was the reduction from baseline in systolic BP (SBP) and diastolic BP (DBP). Results The registry enrolled 315 patients (59.1\% females; mean age $64.84 \pm 12.18$ years). Baseline SBP and DBP were $159.11 \pm 16.93$ and $88.32 \pm 12.35 \mathrm{mmHg}$, respectively. At a mean $2.88 \pm 1.75$ months after starting lercanidipine/enalapril, the mean change from baseline in SBP and DBP were $-18.08 \pm 15.91$ and $-10.10 \pm 11.46 \mathrm{mmHg}$, respectively
\end{abstract}

On behalf of the CONCEPT Collaborative Group.

The participants in the group are given in the Appendix.

J. Maldonado

Instituto de Investigação e Formação Cardiovascular/Clínica da Aveleira, Quinta da Portela, Rua Princesa Sindazunda Lote 9.3/ Loja 94, 3030-503 Coimbra, Portugal

\section{T. Pereira $(\bowtie)$}

Departamento de Cardiopneumologia, Instituto Politécnico de Coimbra, ESTESC, Instituto de Investigação e Formação Cardiovascular, Quinta da Portela, Rua Princesa Sindazunda Lote 9.3/Loja 94, 3030-503 Coimbra, Portugal

e-mail: telmopereira@spc.pt

A. Tavares

Jaba Recordati, S. A., Lagoas Park, Edificio 5, Torre C, Piso 3, 2740-298 Porto Salvo, Portugal (both $p<0.001$ ). This corresponded to reductions of 11.4 and $11.3 \%$ in SBP and DBP, respectively. SBP was reduced independently of sex and age, and DBP was reduced independently of sex. The BP control $(<140 / 90 \mathrm{mmHg})$ rate significantly increased from $10.2 \%$ at baseline to $51.0 \%$ after a mean of 2.88 months of treatment with lercanidipine/enalapril $(p<0.001)$. Adverse effects were seen in only one patient $(0.3 \%)$, who developed a persistent dry cough.

Conclusions Treatment with the fixed-dose combination lercanidipine/enalapril was associated with significant reductions in SBP and DBP, and a significant increase in the BP control rate. This fixed-dose combination has been shown to effectively reduce BP, generally independently of age and sex, and with an excellent safety profile.

\section{Key Points}

This study was an observational registry enrolling 315 patients treated by 46 specialists in hypertension clinics across Portugal. Patients received lercanidipine/enalapril (10/20 mg) fixed-dose combination (FDC) for $\sim 2$ months, and efficacy and safety of the treatment were assessed.

Treatment with lercanidipine/enalapril FDC was associated with significant reductions from baseline in systolic and diastolic blood pressure (BP), and increases in the rate of BP control $(<140 / 90 \mathrm{mmHg})$.

The lercanidipine/enalapril FDC had an excellent safety profile in this population, with treatmentemergent adverse events reported in only one patient.

These results suggest that lercanidipine/enalapril (10/20mg) FDC is an effective and safe treatment for the general hypertensive population in Portugal. 


\section{Introduction}

It is well recognized that arterial hypertension is a leading cause of death and disability worldwide [1]. Hypertension is a significant risk factor for cardiovascular disease, stroke, peripheral vascular disease, and end-stage renal disease [2]. The disease prevalence is impressive, with more than one-quarter of the world's adult population having hypertension at present, and it is expected to increase in future [3].

Reducing blood pressure (BP) has been shown to reduce the risk of hypertension-associated morbidity and mortality [4-6]. However, despite the progressive improvements observed in many countries [7], BP control rates remain suboptimal [8]. Reasons for not achieving BP targets include a lack of adherence to or persistence with antihypertensive therapy, often due to the occurrence of adverse events, the use of drugs that do not target the mechanism(s) of BP elevation in that patient, and monotherapy being insufficient to control BP [9].

Because there are multiple possible mechanisms of BP elevation, and the response to a drug may be attenuated by counter-regulatory responses, two or more antihypertensive drugs of different classes are often required to achieve BP control $[9,10]$. It has been shown that combination therapy using antihypertensive drugs with complementary mechanisms of action has additive BP-lowering effects and is more effective than high-dose monotherapy with the same drugs [11, 12]. Furthermore, because it allows the use of lower doses of each drug than monotherapy, and because in some cases one drug class can attenuate the adverse events that occur with another, combination therapy is likely to be better tolerated $[9,11]$.

A potential disadvantage of combination therapy is the additional pill burden, particularly in patients taking multiple medications for comorbidities. Increasing complexity of dosing has been shown to reduce adherence and persistence with therapy $[10,12,13]$. A strategy to address this problem is the use of fixed-dose combinations (FDCs), which simplifies dosing by allowing two or more drugs to be administered as a single pill. The use of FDCs has been shown to improve adherence to antihypertensive therapy and increase BP control rates $[6,12,14]$. In fact, in some countries, a parallel increase has been noted in BP control rates and the use of combination therapy for the treatment of hypertension $[15,16]$.

There are numerous possible combinations of antihypertensive drugs available as FDCs. The combination of a calcium channel blocker (CCB) and a modulator of the renin-angiotensin system (RAS) appears to be a primary option [6, 17-19]. One such combination is the third-generation vasoselective dihydropyridine $\mathrm{CCB}$ lercanidipine plus the angiotensin-converting enzyme inhibitor (ACEI) enalapril, which is available as an FDC. This combination has been shown to be effective and well tolerated in clinical trials [20-22]. However, there is a lack of data on its efficacy and tolerability in real-world clinical practice, where patients' characteristics are likely to differ from those included in controlled clinical trials. In this context, the CONCEPT Collaborative Group (CCG) aimed to evaluate the efficacy and tolerability of a lercanidipine $10 \mathrm{mg}$ plus enalapril $20 \mathrm{mg}$ FDC in patients with hypertension treated in the non-hospital setting.

\section{Methods}

\subsection{Study Design}

The CCG consists of 46 specialists with a particular interest in cardiovascular diseases (internal medicine and cardiologists) practicing in private clinics in Portugal who decided to perform a critical analysis of their clinical management of private out-of-hospital patients. The CCG established an observational registry to assess the efficacy and safety of lercanidipine/enalapril for the treatment of hypertension. Patient recruitment and assessment took place during a 6-month period.

\subsection{Patients}

All patients with hypertension presenting to a CCG member's clinic who were prescribed lercanidipine/enalapril $(10 / 20 \mathrm{mg})$ were included in the registry. Patients were required to be aged 18 years or older and to have been prescribed the lercanidipine/enalapril FDC as either initial therapy or after previous antihypertensive treatment due to issues of efficacy or tolerability with their existing therapy or because the specialist considered the lercanidipine/ enalapril to be a more suitable treatment than that prescribed by the patient's general practitioner. Patients were initially given lercanidipine/enalapril $10 / 10 \mathrm{mg}$, with the dose increased to $10 / 20 \mathrm{mg}$ from the second clinic visit. Lercanidipine/enalapril 10/20 mg was given either alone or in combination with other antihypertensive drugs in order to achieve a BP target of $<140 / 90 \mathrm{mmHg}$.

\subsection{Assessments}

Data were collected at baseline and after approximately 2 months of treatment with lercanidipine/enalapril $10 / 20 \mathrm{mg}$. At both consultations, the patients' weight and height were measured, and body mass index (BMI) was calculated in $\mathrm{kg} / \mathrm{m}^{2}$. BP was also measured at baseline and 2 months after the patient started treatment with 
lercanidipine/enalapril 10/20 mg. BP measurements were taken in a supine position and after a 10-min resting period by an experienced operator using an oscilometric automatic sphygmomanometer (clinically validated-class A), with appropriate cuff. Before their appointment, patients were advised to avoid coffee or tobacco consumption. Three measurements were taken at each assessment, with a 2-min interval between each measurement, and the arithmetic mean was used in the analysis. Adverse events were collected by the specialists who were instructed to report all situations of interest. For all assessments, a quality check was performed on a regular basis to ensure adequate compliance with all the necessary conditions to warrant the validation of the study.

\subsection{Objectives}

The primary outcome measure was the reduction in systolic and diastolic BP (SBP and DBP, respectively) from baseline after 2 months of treatment with lercanidipine/enalapril 10/20 mg. Secondary endpoints included the proportion of patients achieving BP control, defined as $140 / 90 \mathrm{mmHg}$, the number and classes of concomitant antihypertensive medications at baseline and endpoint (therapeutic profile), and the incidence of treatment-emergent adverse events after starting treatment with lercanidipine/enalapril.

\subsection{Data Management}

All data were codified and personally delivered to the study coordinator (João Maldonado), blinding the name and other means of identifying individual patients. Electronic medical records for individual patients were not obtained by the registry coordinating team. A quality analysis of the data was then performed by the registry coordinators, and all registries with incoherent or incomplete data were excluded.

\subsection{Ethical Considerations}

All procedures followed were in accordance with the ethical standards of the responsible committee on human experimentation (institutional and national) and with the Helsinki Declaration of 1975, as revised in 2000. Informed consent was obtained from all patients included in the registry.

\subsection{Statistical Analysis}

The data were entered into a central database and analyzed using SPSS for Windows, version 17.0. The distribution of the variables was tested for normality using the Shapiro-
Wilk test and for homogeneity of variance by Levene's test. Simple descriptive statistics were used to characterize the sample and the distribution of variables.

Within-group comparisons were made using the chisquared test with Fisher's correction, for categorical variables, the Student's $t$-test for pairwise samples, or the Wilcoxon test for quantitative variables with or without normal distribution.

The criterion for statistical significance used was $p \leq 0.05$ for a confidence interval of $95 \%$.

\section{Results}

\subsection{Baseline Characteristics}

The registry included 315 patients $(59.1 \%$ females) who were treated with lercanidipine/enalapril as first-line therapy or after previous antihypertensive therapy due to lack of efficacy $(n=283)$, adverse events $(n=21)$, or because their physician considered the FDC to be a more suitable treatment than that previously prescribed by the patient's general practitioner $(n=59)$. Many patients switched therapy for more than one reason.

Baseline characteristics are presented in Table 1. The mean age was $64.84 \pm 12.18$ years (range 35-93), and the mean time since the diagnosis of hypertension was $12.28 \pm 13.54$ years. Baseline SBP and DBP were $159.11 \pm 16.93$ and $88.32 \pm 12.35 \mathrm{mmHg}$, respectively. BP was controlled $(<140 / 90 \mathrm{mmHg})$ in $10.2 \%$ of patients. Antihypertensive treatments at baseline are shown in Table 1. The mean number of antihypertensive drugs per patient at baseline was $2.1 \pm 1.3$. The most commonly used antihypertensive classes were diuretics $(45.5 \%$ of patients), ACEIs (40.1\%), angiotensin II receptor antagonists $(33.7 \%)$, $\beta$-blockers (31.9\%), and CCBs (29.3\%). Free combinations were used in $32.2 \%$ of the patients and FDCs in $33.4 \%$.

\subsection{Blood Pressure (BP) Reduction and Control Rates}

BP was measured at a mean of $2.88 \pm 1.75$ months after initiating treatment with lercanidipine/enalapril. Mean changes from baseline for SBP and DBP were $-18.08 \pm$ 15.91 and $-10.10 \pm 11.46 \mathrm{mmHg}$ (Fig. 1; Table 2; $p<0.0001$ for both). This corresponded to mean reductions in SBP and DBP of 11.4 and $11.3 \%$, respectively, compared with baseline. The BP control rate significantly increased from $10.2 \%$ at baseline to $51.0 \%$ after treatment with lercanidipine/enalapril $(p<0.001)$ (Fig. 2). SBP was reduced from baseline, independently of sex and age (Fig. 1), while DBP was reduced independently of sex; patients aged $<60$ years had a significantly greater 
Table 1 Baseline clinical and therapeutic profile of the study population

\begin{tabular}{|c|c|c|c|c|}
\hline & Total $(n=315)$ & Females $(n=186)$ & Males $(n=129)$ & $p$ value \\
\hline Age, years & $64.84 \pm 12.18$ & $65.27 \pm 11.82$ & $64.22 \pm 12.75$ & 0.48 \\
\hline $\mathrm{SBP}, \mathrm{mmHg}$ & $159.11 \pm 16.93$ & $159.64 \pm 16.57$ & $161.18 \pm 16.94$ & 0.45 \\
\hline $\mathrm{DBP}, \mathrm{mmHg}$ & $88.32 \pm 12.35$ & $88.23 \pm 11.79$ & $90.19 \pm 11.58$ & 0.17 \\
\hline $\mathrm{BP}<140 / 90 \mathrm{mmHg}$ & 10.2 & 7.9 & 7.0 & 0.82 \\
\hline$\alpha$-blocker & 1.9 & 2.1 & 1.6 & 0.52 \\
\hline ARAII & 33.7 & 35.4 & 27.1 & 0.06 \\
\hline$\beta$-blocker & 31.9 & 30.8 & 32.9 & 0.38 \\
\hline $\mathrm{CCB}$ & 29.3 & 30.9 & 28.7 & 0.42 \\
\hline ACEI & 40.1 & 42.1 & 39.7 & 0.50 \\
\hline Diuretic & 45.5 & 49.4 & 31.8 & 0.01 \\
\hline Renin inhibitor & 5.4 & 5.9 & 4.6 & 0.40 \\
\hline Free combination & 32.2 & 34.6 & 20.2 & 0.23 \\
\hline Fixed-dose combination & 33.4 & 34.5 & 25.6 & 0.05 \\
\hline Number of antihypertensive drugs & $2.1 \pm 1.3$ & $2.09 \pm 1.24$ & $1.71 \pm 1.26$ & 0.06 \\
\hline
\end{tabular}

All values are mean $\pm \mathrm{SD}$ or $\%$ of patients, unless otherwise stated

$A C E I$ angiotensin-converting enzyme inhibitor, $A R A I I$ angiotensin II receptor antagonist, $B P$ blood pressure, $C C B$ calcium-channel blocker, $D B P$ diastolic blood pressure, pts patients, $S B P$ systolic blood pressure, $S D$ standard deviation

Fig. 1 Blood pressure reduction after adding lercanidipine/enalapril $10 / 20 \mathrm{mg}$ fixed-dose combination; overall population, and stratified according to sex and age. $* p=0.001$ versus DBP reduction in patients aged $\geq 60$ years. $B P$ blood pressure, $D B P$ diastolic blood pressure, $S B P$ systolic blood pressure

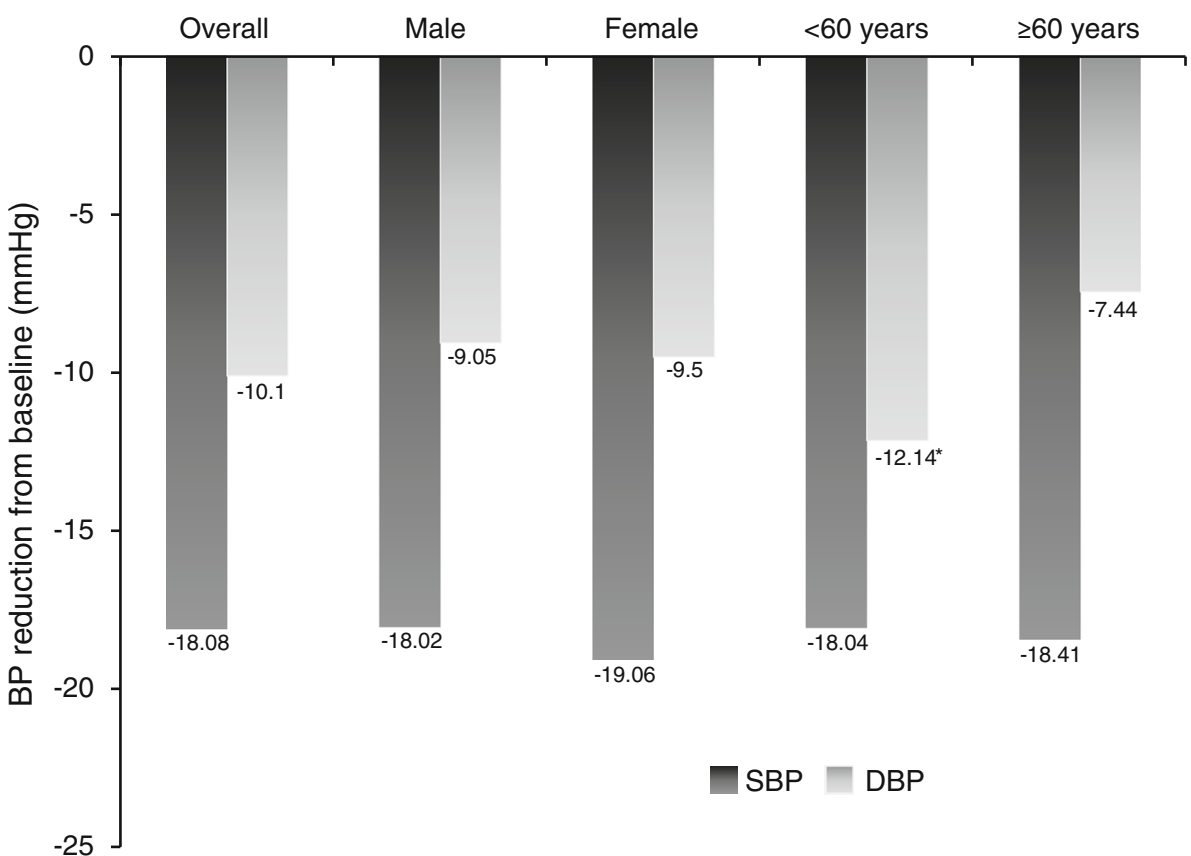

reduction from baseline in DBP than patients aged $\geq 60$ years ( $p=0.001$; Fig. 1$)$. BP control rates in the analysis by age were similar to those of the overall population; control rates before and after treatment in patients aged $<60$ years were 4.3 and $51.1 \%$, while those in patients aged $\geq 61$ years were 8.7 and $50 \%$.

This effect was observed irrespective of whether or not patients were receiving concomitant antihypertensive treatment; however, the magnitude of the BP reduction observed was greater in patients receiving lercanidipine/ enalapril alone compared with patients receiving the FDC with other antihypertensive drugs (Table 3 ). These differences may arise from the fact that patients who received the FDC alone had higher baseline BP and lower baseline $\mathrm{BP}$ control rates (despite the fact that all patients who received FDC alone were not antihypertensive treatment naïve) than those who received the FDC with other antihypertensive drugs (1.9 vs. $11.8 \%$, respectively; $p=0.033)$. By $\sim 2$ months of treatment with lercanidipine/enalapril, the BP levels were similar between patients 
Table 2 Blood pressure levels before and after adding lercanidipine/enalapril fixed-dose combination

\begin{tabular}{lcccc}
\hline & Baseline & After adding FDC & Mean difference $(95 \%$ CI $)$ & $p$ value \\
\hline Mean SBP, mmHg & $159.11 \pm 16.93$ & $141.04 \pm 14.60$ & $-18.08 \pm 15.91(-19.84,-16.31)$ & $<0.0001$ \\
Mean DBP, mmHg & $88.32 \pm 12.35$ & $78.22 \pm 11.86$ & $-10.10 \pm 11.46(-11.37,-8.83)$ & $<0.0001$ \\
\hline
\end{tabular}

All values are mean \pm SD unless otherwise stated

$C I$ confidence interval, $D B P$ diastolic blood pressure, $F D C$ fixed-dose combination, $S B P$ systolic blood pressure, $S D$ standard deviation
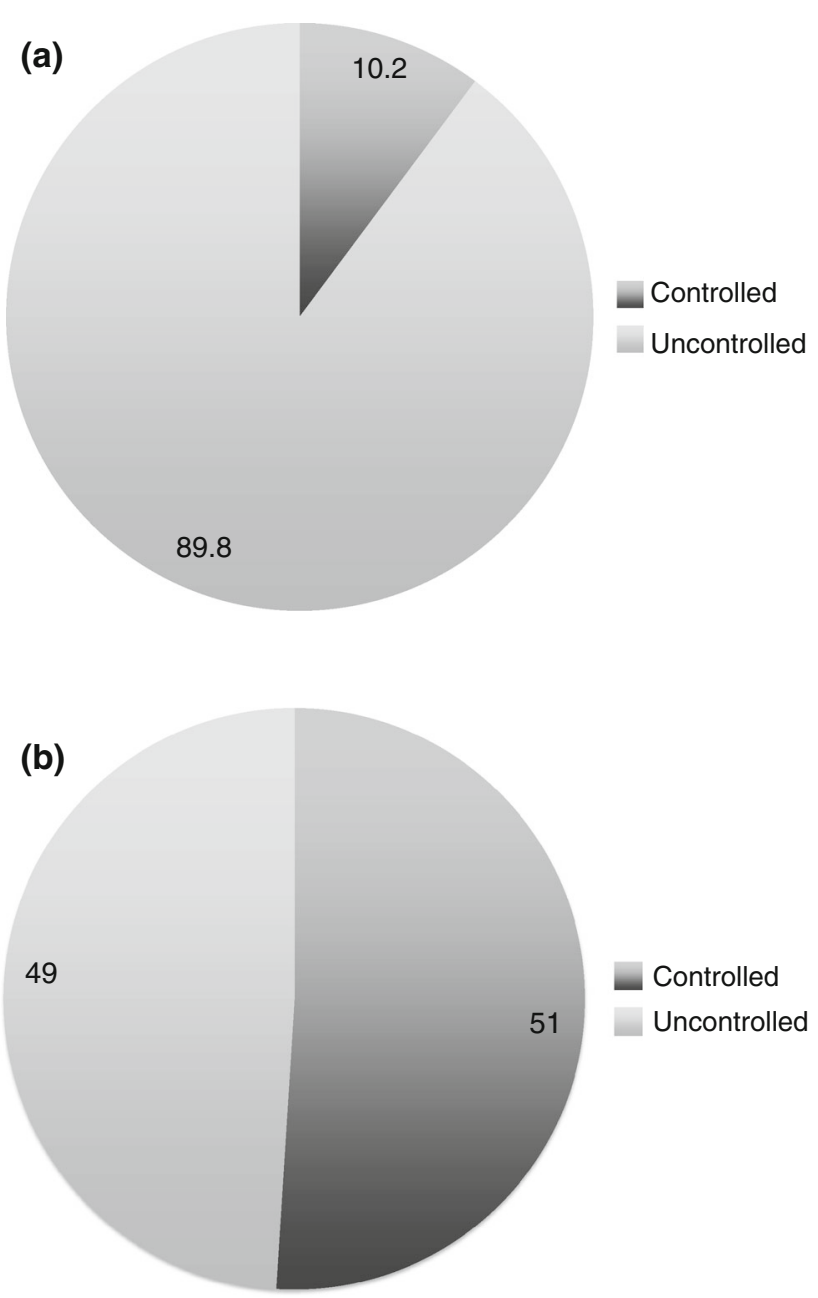

Fig. 2 Blood pressure control rate (a) before (baseline) and (b) after adding lercanidipine/enalapril 10/20 mg fixed-dose combination

receiving the FDC alone and patients receiving the FDC with other antihypertensive drugs (141.16 \pm 15.06 vs. $140.38 \pm 12.10$ for SBP; $78.03 \pm 12.45$ vs. $79.15 \pm 8.31$ for DBP), as were the control rates (51.5 and $48.1 \%$ ).

The magnitude of the BP response was slightly greater in patients not previously treated with ACEIs and/or CCBs, as expected, although BP significantly reduced in both conditions (Table 4). Baseline and post-lercanidipine/enalapril BP levels were similar in both cases.

Finally, there were no significant differences between the number of concomitant drugs received between the age groups, although a trend for a lower number was seen in the younger group (1.7 vs. 2.0, $p=$ not significant).

\subsection{Therapeutic Profile}

The use of most other classes of antihypertensive medication decreased slightly from baseline after starting treatment with lercanidipine/enalapril; only the proportion of patients receiving an $\alpha$-blocker (2.2\%) was higher than at baseline (Fig. 3). All patients were given lercanidipine/ enalapril, and $23.3 \%$ were taking a free combination regimen; none of the patients received an FDC other than lercanidipine/enalapril. No patients switched to lercanidipine + enalapril as a free combination. The mean number of antihypertensive drugs per patient increased to $2.8 \pm 0.9$ at a mean of 2.88 months after addition of lercanidipine/ enalapril, although the difference from baseline was not statistically significant $(p=0.321)$.

\subsection{Tolerability}

Treatment with lercanidipine/enalapril was well tolerated. Treatment-emergent adverse effects occurred in only one patient $(0.3 \%)$, who developed a persistent dry cough after the initiation of lercanidipine/enalapril treatment. This cough was considered to be possibly related to treatment with enalapril. None of the patients developed edema.

\section{Discussion}

This observational registry study showed that treatment with a lercanidipine/enalapril FDC was associated with significant reductions in SBP and DBP and a significant increase in the proportion of patients achieving BP control compared with baseline.

The reduction in BP observed in our study was as expected with combinations of two or more antihypertensive drugs. A meta-analysis by Law et al. [11] found that the use of two antihypertensive drugs at half-standard doses produced reductions in SBP and DBP of 13.3 and $7.3 \mathrm{mmHg}$, respectively; corresponding values for three drugs at half-standard doses were 19.9 and $10.7 \mathrm{mmHg}$, respectively11. Our results are also in agreement with the well known efficacy of an FDC of a CCB with a modulator 
Table 3 Change in blood pressure levels in patients who received lercanidipine/enalapril fixed-dose combination alone and those who received the lercanidipine/enalapril in combination with other antihypertensive drugs

\begin{tabular}{llll}
\hline Change from baseline & Lercanidipine/enalapril alone $(n=52)$ & Lercanidipine/enalapril + antihypertensives $(n=262)$ & $p$ value \\
\hline Mean SBP, mmHg & $-28.52 \pm 15.00$ & $-16.00 \pm 15.28$ & $<0.0001$ \\
Mean DBP, mmHg & $-9.36 \pm 11.89$ & $-13.79 \pm 8.05$ & 0.01 \\
\hline
\end{tabular}

All values are mean \pm SD unless otherwise stated

$D B P$ diastolic blood pressure, $S B P$ systolic blood pressure

Table 4 Change in blood pressure levels with lercanidipine/enalapril fixed-dose combination treatment in patients who were receiving angiotensin-converting enzyme inhibitor and/or calcium-channel blocker treatment at baseline compared with patients who were not

\begin{tabular}{lcc}
\hline Change from baseline with lercanidipine/enalapril treatment & Previous ACEI and/or CCB & No previous ACEI/CCB \\
\hline Mean SBP, mmHg & $-16.33 \pm 15.73$ & $-20.11 \pm 15.93$ \\
Mean DBP, mmHg & $-8.41 \pm 10.73$ & $-12.06 \pm 11.99$
\end{tabular}

All values are mean \pm SD unless otherwise stated

$A C E I$ angiotensin-converting enzyme inhibitor, $C C B$ calcium-channel blocker, $D B P$ diastolic blood pressure, $S B P$ systolic blood pressure, $S D$ standard deviation

Fig. 3 Therapeutic profile before (baseline) and after adding lercanidipine/enalapril $10 / 20 \mathrm{mg}$ fixed-dose converting enzyme inhibitor, $A R A I I$ angiotensin II receptor antagonist, $C C B$ calcium channel blocker, $F D C$ fixeddose combination, $R I$ renin inhibitor combination. ACEI angiotensin-

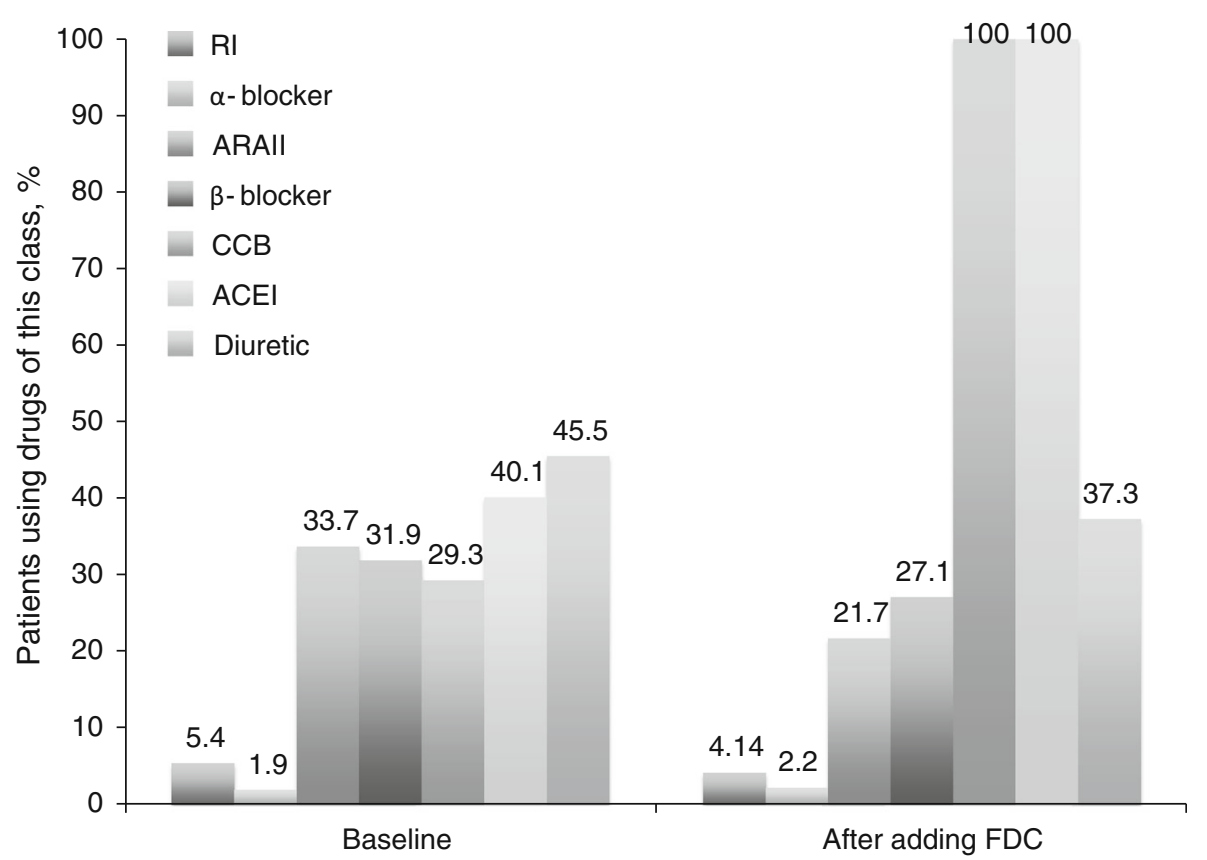

of the RAS [20], even if we consider the relatively old population evaluated, and the extended period of treatment between diagnosis and inclusion in this study. In this context, the rate of $\mathrm{BP}$ control was also impressive, being observed in $51 \%$ of patients with $\mathrm{BP}<140 / 90 \mathrm{mmHg}$ after a mean of 2.88 months of treatment with the fixed-dose regimen.

In randomized, controlled phase III trials of lercanidipine/enalapril FDC, reductions in SBP and DBP of 7.7-9.8 and $7.1-9.2 \mathrm{mmHg}$, respectively, were observed after 12 weeks of treatment [21]. The reductions in SBP and
DBP observed in our study were greater than this (18.08 and $10.10 \mathrm{mmHg}$, respectively). In these two studies, the proportion of patients with normalized SBP and DBP was $22-24 \%$ [21]. It should be noted that these studies included only patients who had not achieved BP control with either lercanidipine or enalapril as monotherapy, and this could have contributed to the smaller reductions in BP and lower BP control rates compared with our study. Furthermore, one of these studies used a lower dose of enalapril $(10 \mathrm{mg})$ than in our study and produced smaller reductions in SBP and DBP than seen with lercanidipine/enalapril 
$10 / 20 \mathrm{mg}$ in the second study. It should also be noted that the patients included in our registry had been receiving antihypertensive regimens prescribed by general practitioners rather than specialists. It is therefore possible that even where their initial therapy had shown BP-lowering activity it may have been suboptimal, and thus further reduction in BP could be obtained by switching to a more suitable therapy, in this case the lercanidipine/enalapril FDC.

The population of our registry was relatively old (mean age approximately 65 years). The age of the study population may have meant that there was a higher proportion of patients with isolated systolic hypertension (ISH) than would have been seen for a study with a younger population. However, baseline BP measurements were averaged, so it was not possible to determine the proportion of patients with ISH. Patients with ISH have marked arterial stiffening, which makes BP control more difficult. In light of the possibility that a significant proportion of patients in our study could have had ISH, the BP-lowering and BP control rates observed are even more impressive. Our results are comparable to those seen in a study in elderly patients (age 60-85 years), in which treatment with the combination of lercanidipine $10 \mathrm{mg}$ plus enalapril $20 \mathrm{mg}$ for 4 weeks was associated with a reduction in SBP of $16.9 \mathrm{mmHg}$ compared with baseline, and a BP control rate of $45 \%$ [20].

In this study, the BP-reducing effect of lercanidipine/ enalapril was greater in patients receiving lercanidipine/ enalapril alone compared with patients receiving the FDC with other antihypertensive drugs. However, at the end of the study period, the mean BP values and BP control rates in both patient groups were similar. This can best be explained by the fact that the magnitude of the therapeutic benefit is generally correlated with baseline BP values [22]. As the patients who received lercanidipine/enalapril alone had significantly greater baseline BP values and lower BP control rates than those who received lercanidipine/enalapril with other antihypertensive drugs, the greater magnitude of improvement at the end of the study in patients who received lercanidipine/enalapril alone was expected.

The introduction of this FDC, in addition to the noted efficacy, did not significantly increase the number of drugs required to achieve BP control. These results may be particularly interesting from an economic perspective, as a reduction in the number of concomitant medications has the potential to produce cost savings, particularly for a high-prevalence disease such as hypertension.

The primary limitation of this study was that it was an open-label pharmaco-epidemiological registry, with all the inherent limitations and advantages of such a design. Other limitations were the relatively small number of patients and the short follow-up duration. The size of the study was necessarily limited by the number of patients presenting to
CCG members' clinics during the study period for whom the lercanidipine/enalapril (10/20 mg) FDC was considered the most appropriate treatment.

Finally, the extremely low incidence of adverse effects noted after initiating treatment with the lercanidipine/ enalapril FDC was especially interesting. Despite the excellent tolerability attributed to the new dihydropyridines, namely with respect to the incidence of ankle edema [23, 24], it may be surprising that none of the patients developed edema with lercanidipine in this study. However, the combination of a CCB with a modulator of the RAS has been shown to reduce the incidence of such events, through a well established mechanism [21, 25]. Only a single case of cough was reported in our study, and this was considered to be possibly related to enalapril as cough is a known adverse effect of ACEIs [26]. Cough was the most common adverse event observed in clinical trials of lercanidipine/enalapril FDC [21]. The incidence of peripheral edema with the FDC also appears to be low, with only $1.5 \%$ of patients treated with lercanidipine/ enalapril $10 / 20 \mathrm{mg}$ for up to 52 weeks in clinical trials experiencing this adverse event [21].

\section{Conclusion}

Treatment with an FDC of lercanidipine/enalapril (10/ $20 \mathrm{mg}$ ) for a mean of 2.88 months was associated with a significant reduction of SBP and DBP and an increase in the $\mathrm{BP}$ control rate from 10.2 to $51.0 \%$, relative to baseline, a result achieved with a reduction in the number of drugs used. The lercanidipine/enalapril FDC was shown to effectively reduce BP, generally independently of age and sex, and with an excellent safety profile.

Acknowledgments This registry was funded by an operational grant from Jaba Recordati S.A., Portugal.

Medical writing assistance was provided by Raewyn Poole, on behalf of inScience Communications, Springer Healthcare. This assistance was funded by Jaba Recordati S.A., Portugal.

Authors' conflict of interests João Maldonado declares that he has no conflict of interest. Telmo Pereira declares that he has no conflict of interest. Alfredo Tavares is an employee of Jaba Recordati S.A.

Open Access This article is distributed under the terms of the Creative Commons Attribution Noncommercial License which permits any noncommercial use, distribution, and reproduction in any medium, provided the original author(s) and the source are credited.

\section{Appendix: Participants in the CONCEPT Collaborative Group}

This registry is the result of the commitment and dedication of a group of 46 specialists with a particular interest in 
cardiovascular diseases, listed below. Paula Gago, Idalécio Bernardo, Pedro Miguel Balza, Sanjiva Cadocar, Nuno Jorge Fonseca, Filipe Seixo, Lurdes Almeida, Marco Aurélio Castro, Pedro Silva Cunha, Hugo Filipe Pego, Fátima Veiga, Luís Filipe Pereira, Susana Castela, Carvalho Rodrigues, João Maria Abecassis, Susana Martins, Graça Almeida, Omar Zalueta Pereira, Paulo Ramos, João Madeira Lopes, Sílvio Leal, Carlos Aguiar, Pedro Von Haffe, Maria José Ferreira, Cristina Rodrigues, Isabel Maria Vilaça, Emília Barbosa, Abílio Ribeiro, Gonçalo Rocha, Sérgio Miguel Silva, Manuel Pinto Monteiro, Fernando Santos Reis, José Bernardes Correia, João Porto, Ana Sofia Teixeira, Rui Providência, José Alexandre Antunes, Rui Pires, António Antunes, Leonel Pinto, João Miguel Santos, João Maldonado, André Paupério, Meireles Brandão, Mário Almeida, Pedro Semedo.

\section{References}

1. World Health Organization. Global health risks: mortality and burden of disease attributable to selected major risks. 2009. http:// www.who.int/healthinfo/global_burden_disease/GlobalHealth Risks_report_full.pdf. Accessed 31 May 2013.

2. Chobanian AV, Bakris GL, Black HR, Cushman WC, Green LA, Izzo JL Jr, et al. Seventh report of the Joint National Committee on Prevention, Detection, Evaluation, and Treatment of High Blood Pressure. Hypertension. 2003;42:1206-52.

3. Kearney PM, Whelton M, Reynolds K, Muntner P, Whelton PK, He J. Global burden of hypertension: analysis of worldwide data. Lancet. 2005;365:217-23.

4. MacMahon S, Peto R, Cutler J, Collins R, Sorlie P, Neaton J, et al. Blood pressure, stroke, and coronary heart disease. Part 1, Prolonged differences in blood pressure: prospective observational studies corrected for the regression dilution bias. Lancet. 1990;335:765-74.

5. Lewington S, Clarke R, Qizilbash N, Peto R, Collins R. Prospective studies C. Age-specific relevance of usual blood pressure to vascular mortality: a meta-analysis of individual data for one million adults in 61 prospective studies. Lancet. 2002;360: 1903-13.

6. Mancia G, Fagard R, Narkiewicz K, Redón J, Zanchetti A, Böhm $\mathrm{M}$, et al. $2013 \mathrm{ESH} / \mathrm{ESC}$ Guidelines for the management of arterial hypertension: The Task Force for the management of arterial hypertension of the European Society of Hypertension (ESH) and of the European Society of Cardiology (ESC). Eur Heart J. 2013;31:1281-357.

7. Kearney PM, Whelton M, Reynolds K, Whelton PK, He J. Worldwide prevalence of hypertension: a systematic review. J Hypertens. 2004;22:11-9.

8. Chow CK, Teo KK, Rangarajan S, Islam S, Gupta R, Avezum A, et al. Prevalence, awareness, treatment, and control of hypertension in rural and urban communities in high-, middle-, and low-income countries. JAMA. 2013;310:959-68.

9. Gradman AH, Basile JN, Carter BL, Bakris GL, Materson BJ, Black HR, et al. Combination therapy in hypertension. J Am Soc Hypertens. 2010;4:90-8.
10. Dusing R. Optimizing blood pressure control through the use of fixed combinations. Vasc Health Risk Manag. 2010;6:321-5.

11. Law MR, Wald NJ, Morris JK, Jordan RE. Value of low dose combination treatment with blood pressure lowering drugs: analysis of 354 randomised trials. BMJ. 2003;326:1427.

12. Gupta AK, Arshad S, Poulter NR. Compliance, safety, and effectiveness of fixed-dose combinations of antihypertensive agents: a meta-analysis. Hypertension. 2010;55:399-407.

13. Bangalore S, Kamalakkannan G, Parkar S, Messerli FH. Fixeddose combinations improve medication compliance: a metaanalysis. Am J Med. 2007;120:713-9.

14. Mancia G, Laurent S, Agabiti-Rosei E, Ambrosioni E, Burnier M, Caulfield MJ, et al. Reappraisal of European guidelines on hypertension management: a European Society of Hypertension Task Force document. J Hypertens. 2009;27:2121-58.

15. Coca A. Evolucion del control de la hipertension arterial en atencion primaria en Espana. Resultados del estudio Controlpress 2003. Hipertension. 2005;22:5-14.

16. Sociedade Portuguesa de Hipertensao. Prevalencia da hipertensao arterial e consumo de sal em Portugal. Rev Port Hipertensao e Risco Cardiovascular. 2013;34:8-9.

17. Bakris G, Molitch M, Hewkin A, Kipnes M, Sarafidis P, Fakouhi $\mathrm{K}$, et al. Differences in glucose tolerance between fixed-dose antihypertensive drug combinations in people with metabolic syndrome. Diabetes Care. 2006;29:2592-7.

18. Jamerson K, Weber MA, Bakris GL, Dahlof B, Pitt B, Shi V, et al. Benazepril plus amlodipine or hydrochlorothiazide for hypertension in high-risk patients. N Engl J Med. 2008;359: 2417-28.

19. Matsui Y, Eguchi K, O’Rourke MF, Ishikawa J, Miyashita H, Shimada K, et al. Differential effects between a calcium channel blocker and a diuretic when used in combination with angiotensin II receptor blocker on central aortic pressure in hypertensive patients. Hypertension. 2009;54:716-23.

20. Puig JG, Calvo C, Luurila O, Luurila H, Sulosaari S, Strandberg A, et al. Lercanidipine, enalapril and their combination in the treatment of elderly hypertensive patients: placebo-controlled, randomized, crossover study with four ABPM. J Hum Hypertens. 2007;21:917-24.

21. Hair PI, Scott LJ, Perry CM. Fixed-dose combination lercanidipine/enalapril. Drugs. 2007;67:95-106.

22. Currie CJ, Peters JR, Tynan A, Evans M, Heine RJ, Bracco OL, et al. Survival as a function of $\mathrm{HbA1c}$ in people with type 2 diabetes: a retrospective cohort study. Lancet. 2010;375:481-9.

23. Makani H, Bangalore S, Romero J, Htyte N, Berrios RS, Makwana $\mathrm{H}$, et al. Peripheral edema associated with calcium channel blockers: incidence and withdrawal rate-a meta-analysis of randomized trials. J Hypertens. 2011;29:1270-80.

24. Makani H, Bangalore S, Romero J, Wever-Pinzon O, Messerli FH. Effect of renin-angiotensin system blockade on calcium channel blocker-associated peripheral edema. Am J Med. 2011; 124:128-35.

25. Messerli FH, Oparil S, Feng Z. Comparison of efficacy and side effects of combination therapy of angiotensin-converting enzyme inhibitor (benazepril) with calcium antagonist (either nifedipine or amlodipine) versus high-dose calcium antagonist monotherapy for systemic hypertension. Am J Cardiol. 2000;86:1182-7.

26. Izzo JL Jr, Weir MR. Angiotensin-converting enzyme inhibitors. J Clin Hypertens. 2011;13:667-75. 